\title{
Liposomal Encapsulation of Oleuropein and an Olive Leaf Extract: Molecular Interactions, Antioxidant Effects and Applications in Model Food Systems
}

\author{
Rodrigo González-Ortega ${ }^{1}$ - Luka Šturm ${ }^{1}$ - Mihaela Skrt ${ }^{1}$ - Carla Daniela Di Mattia ${ }^{2}$ Paola Pittia ${ }^{2}$. \\ Nataša Poklar Ulrih ${ }^{1,3}$
}

Received: 4 June 2020 / Accepted: 26 August 2020 / Published online: 2 October 2020

(C) The Author(s) 2020

\begin{abstract}
The influence of actively/passively encapsulated oleuropein on DPPC liposomes thermal and structural properties, and its antioxidant capacity against lipid peroxidation were investigated. Also, an oleuropein-rich olive leaf extract was encapsulated in soy phosphatidylcholine (PL-90 g) and incorporated in model and commercial drinks. Oleuropein induced a concentrationdependent broadening and splitting of the gel-to-liquid phase transition temperature. Fluorescence measurements revealed a fluidizing effect on liposomes below their gel-to-liquid phase transition temperature, and a higher lipid ordering above, especially to active encapsulation. Oleuropein also showed an antioxidant effect against lipid peroxidation in PL-90 g liposomes. PL-90 g Liposomes with olive leaf extract showed a mean diameter of $405 \pm 4 \mathrm{~nm}$ and oleuropein encapsulation efficiency of $34 \%$ and delayed oleuropein degradation at $\mathrm{pH} 2.0$ and 2.8 model drinks. In conclusion, greater effects were observed on the structure and fluidity of DPPC liposomes when oleuropein was actively encapsulated, while its incorporation into acidic foods in encapsulated form could enhance its stability.
\end{abstract}

Keywords Encapsulation $\cdot$ Liposomes $\cdot$ Oleuropein $\cdot$ Olive leaf extract $\cdot$ Phospholipid membranes $\cdot$ Peroxidation

\section{Introduction}

Oleuropein is the most abundant biophenol in the olive tree fruits and leaves. It is an ester of hydroxytyrosol and a glucoside derivative of elenolic acid. It has been shown to have powerful biological properties that have been attributed to

Electronic supplementary material The online version of this article (https://doi.org/10.1007/s11483-020-09650-y) contains supplementary material, which is available to authorized users.

Nataša Poklar Ulrih

natasa.poklar@bf.uni-lj.si

1 Department of Food Science and Technology, Biotechnical Faculty, University of Ljubljana, Jamnikarjeva 101, Ljubljana SI-1000, Slovenia

2 Present address: Faculty of Bioscience and Technology for Food Agriculture and Environment, University of Teramo, Via Renato Balzarini 1, 64100 Teramo, Italy

3 The Centre of Excellence for Integrated Approaches in Chemistry and Biology of Proteins (CipKeBiP), Jamova 39,

SI-1000 Ljubljana, Slovenia the hydroxytyrosol moiety. These include radical scavenging [1-3] and antimicrobial activity [4, 5], with inhibition of LDL peroxidation and platelet aggregation involved in prevention of diseases like atherosclerosis [6,7] and cancers [8]. For these reasons, and along with many other biophenols, oleuropein has attracted interest in the food industry for formulation of functional products with increased nutritional properties, or with enhanced shelf-life through exploitation of its antimicrobial properties and inhibition of food oxidation [9].

However, the use of such single compounds or extracts from natural raw materials in foods, pharmaceutics and cosmetics is often challenging, due to their low solubilities, sensitivities to environmental conditions (e.g., temperature, $\mathrm{pH}$, light), and often poor sensorial characteristics. For example, oleuropein is a bitter compound that is generally broken down into the bland tasting hydroxytyrosol and elenolic acid at acidic and alkaline pHs during table olive production [10]. However, there is good evidence that oleuropein might be the most suitable precursor of hydroxytyrosol and other bioactive metabolites for food and nutraceuticals. Oleuropein appears to have higher bioavailability when it reaches the colon, where it is fermented by the microflora, to result in a large array of bioactive compounds [11, 12]. 
To overcome these problems of the biophenolics, different techniques of micro-encapsulation and nano-encapsulation have been developed over the last few decades, to enhance their usability and bioavailability [13]. Liposomes are vesicles that are composed of one or more phospholipid bilayers, and they have been widely used in the pharmaceutical and cosmetic industries. They have also shown interesting characteristics for the food industry; e.g., entrapment of water-soluble, lipid-soluble and amphiphilic compounds, slow release, and the possibility for largescale production [14]. The most common and up-scalable techniques for liposome preparation include thin-film formation and the proliposome method, which result in the formation of liposomes of differing sizes and degrees of lamellarity (multilamellar vesicles). ). Liposomes with the correct qualities, such as high encapsulation efficiency, good stability, narrow particle size, and controlled release, have been designed to encapsulate food bioactives using the proliposome method $[15,16]$.

Liposomes are also a widely used model to evaluate interactions with and effects of antioxidants on biological membranes, and this information is also useful to engineer and interpret the behavior and dynamics of liposomes as carriers in food systems [14, 17]. Several studies have evaluated oleuropein and other olive biophenols for their interactions with and effects on phospholipid membranes, in terms of lipid ordering, fluidity and free-radical scavenging. Some studies showed the antioxidant activity of oleuropein against lipid peroxidation and the positioning of oleuropein deep within membranes [18]. On the other hand, more recent studies showed shallower positioning within membranes and radical scavenging activity of oleuropein near to the polar headgroups of the phospholipids and the aqueous phase, with these related to the nature and polarity of the phospholipid headgroups [19, 20]. However, there is no information in the literature related to the impact of encapsulation of oleuropein on membrane physicochemical properties, as the majority of these studies have used titration of liposome suspensions with the bioactive compound of interest, and subsequent partitioning.

In the present study, model liposomes were used to investigate the effects of oleuropein on membrane thermotropic behavior (differential scanning calorimetry [DSC]) and ordering and fluidity (fluorescence polarization) in systems with passively encapsulated oleuropein (i.e., added after formation of liposomes) compared to actively encapsulated oleuropein (i.e., encapsulated during formation of liposomes). Also, the antioxidant capacity was evaluated under two types of oxidation induction, in terms of oleuropein inhibition of lipid peroxidation. Finally, an oleuropein-rich olive leaf extract (OLE) was actively encapsulated in liposomes using the proliposome method with commercial soy phosphatidylcholine (PL-90 g). The morphological and physicochemical properties of these liposomes with actively encapsulated oleuropein were determined, along with the oleuropein stability under different $\mathrm{pH}$ conditions in a food model and a real food system.

\section{Materials and methods}

\section{Materials and chemicals}

The standardized OLE powder was kindly provided by Oleafit S.r.l (Isola del Gran Sasso, Italy). This OLE was obtained from leaves of the olive tree Olea europaeia L by maceration in 70\% aqueous ethanol. According to the manufacturer specifications, OLE contains a minimum of $40 \mathrm{wt} \%$ (dry weight) oleuropein, $3.15 \mathrm{wt} \%$ ash, and $3.22 \mathrm{wt} \%$ moisture. Phospholipon $90 \mathrm{~g}$ (PL-90 g; commercial lipid mixture which contains pure phosphatidylcholine 94.0-102.0\% from soybean; lysophosphatidylcholine $4 \%$; tocopherol $0.3 \%$ stabilized with ascorbyl palmitate $0.1 \%$ ) was from Phospholipid GmbH (Köln, Germany). 1,2-Dipalmitoyl-sn-glycero-3phosphocholine (DPPC, molecular weight, $\mathrm{MW}=734 \mathrm{~g} \mathrm{~mol}^{-}$

${ }^{1}$ ) was from Avanti Polar Lipids (Alabaster, AL). Commercial lemonade nectar was purchased in a local supermarket (Sola limonada; Sola, Pivovarna Lasko Union d.o.o, Slovenia). Oleuropein ( $\geq 80 \%$ by HPLC), L-ascorbic acid, and the fluorophores 1,6-diphenyl-1,3,5-hexatriene (DPH) and N,N,N-trimethyl-4-(6-phenyl-1,3,5-hexatrien-1-yl) phenylammonnium p-toluenesulfonate (TMA-DPH) were from Sigma-Aldrich (Darmstadt, Germany). Hydroxytyrosol ( $\geq 98 \%$ by HPLC) and verbascoside ( $\geq 99 \%$ by HPLC) were from Extrasynthese (Lyon, France). The fluorescent probe 4,4-difluoro-5-(4-phenyl-1,3-butadienyl)-4-bora-3a,4a-diazas-indacene-3-undecanoic acid (BODIPY 581/591 C11) was from Molecular Probes (Eugene, OR), and thiobarbituric acid was from Merck (Darmstadt, Germany). All of the other chemicals used were of analytical reagent grade, and all aqueous solutions were prepared with ultrapure water (RIOS 5 purification system; Millipore, Bedford, MA).

\section{High-performance liquid chromatography}

The oleuropein concentrations were determined by HPLC (1260 Infinity system; Agilent Technologies, Santa Clara, CA) using a C18 column (Zorbax Eclipse Plus; $4.6 \times$ $150 \mathrm{~mm} ; 3.5 \mu \mathrm{m}$; Agilent Technologies) with a $\mathrm{C} 18$ analytical guard column (Eclipse XDB-C18; $4.6 \times 12.5 \mathrm{~mm} ; 5 \mu \mathrm{m}$; Agilent Technologies). The elution conditions were: column temperature, $25{ }^{\circ} \mathrm{C}$; injection volume, $20 \mu \mathrm{l}$; and flow rate, $0.3 \mathrm{~mL} \mathrm{~min}^{-1}$. The mobile phases consisted of $1 \%(\mathrm{v} / \mathrm{v})$ acetic acid (A) and acetonitrile (B), with the separation carried out with the following gradient: $0-16 \mathrm{~min}, 5 \%-15 \% \mathrm{~B} ; 16-$ $37 \mathrm{~min}, 15 \%-30 \% \mathrm{~B}$; 37-50 min, 30\%-40\%; 50-58 min, 40\%-50\% B; 58-60 min, 50\%-100\% B; 60-61 min, $100 \%$ B; 61-62 min, $100 \%-5 \% \mathrm{~B}$; re-equilibration from 62 to $70 \mathrm{~min}, 5 \% \mathrm{~B}$. The chromatograms were monitored at $360 \mathrm{~nm}$ for verbascoside and $280 \mathrm{~nm}$ for oleuropein and hydroxytyrosol. Calibrations curves for the oleuropein, verbascoside, and hydroxytyrosol concentrations were 
prepared using standards at $10-200 \mu \mathrm{g} \mathrm{mL}^{-1}, 5-80 \mu \mathrm{g} \mathrm{mL}^{-1}$ and $5-80 \mu \mathrm{g} \mathrm{mL}^{-1}$, respectively. To characterize the phenolic composition of the OLE, it was dissolved in aqueous ethanol $(1: 1 ; \mathrm{v} / \mathrm{v})$, which was centrifuged $(10,000 \times g, 5 \mathrm{~min})$ and filtered $(0.2 \mu \mathrm{m}$ nylon syringe filter) before HPLC analysis.

\section{Preparation of liposomes}

The multilamellar liposomes used for the oleuropeinphospholipid membrane interactions and for lipid peroxidation were prepared with DPPC and PL-90 g, respectively, using the thin-layer film method [21]. Briefly, DPPC or PL$90 \mathrm{~g}$ were dissolved in chloroform:methanol $(2: 1, \mathrm{v} / \mathrm{v})$ in a round-bottomed flask, without oleuropein (for later passive encapsulation of oleuropein) and with oleuropein (for active encapsulation). The solvents were slowly and completely evaporated under vacuum $(<20 \mathrm{mbar})$ at $35^{\circ} \mathrm{C}$ in a rotatory evaporator (Rotavapor R-210; Büchi, Labortechnik AG, Switzerland). The resulting thin phospholipid films were hydrated in $20 \mathrm{mM}$ HEPES (pH 7.0) under shaking at $50^{\circ} \mathrm{C}$ for $1 \mathrm{~h}$, to a final lipid concentration of $5 \mathrm{mg} \mathrm{mL}^{-1}$ DPPC (6.81 mM, MW = $734 \mathrm{~g} \mathrm{~mol}^{-1}$ ) or $14 \mathrm{mg} \mathrm{mL}^{-1}$ PL-90 g (18.46 mM, considering the molecular weight of the major phospholipid in soybean, 1-palmitoyl-2-linoleoyl-sn-glycero3-phosphocholine, equal to $758.1 \mathrm{~g} \mathrm{~mol}^{-1}$, see [22]). The DPPC liposome suspensions with actively encapsulated oleuropein (two molar ratios of oleuropein:DPPC, $n_{\text {Oleu }}$ / $n_{\text {DPPC }}$, of 1:5 and 1:1 were prepared) were used directly, and those without oleuropein (DPPC only) were prepared and then titrated with increasing concentrations of oleuropein, to provide the liposomes with passively encapsulated oleuropein.

The DPPC liposomes, used for DSC and fluorescence polarization measurements, were diluted before analysis in oleuropein solutions in $20 \mathrm{mM}$ HEPES ( $\mathrm{pH}$ 7.0) (for liposomes with passive encapsulated oleuropein) or in $20 \mathrm{mM}$ HEPES ( $\mathrm{pH}$ 7.0) (for liposomes with actively encapsulated oleuropein), to the final lipid concentration of $1.36 \mathrm{mM}$ (1 $\mathrm{mg} \mathrm{mL}^{-1}$ ).

For the fluorescence emission polarization and BODIPY fluorescence assays, the DPPC and PL-90 g liposomes were used to prepare SUVs. This was achieved by high intensity ultrasonication (amplitude, 40\%; power output, $750 \mathrm{~W}$; Vibra Cell VCX 750; Sonics) for $15 \mathrm{~min}$, as intervals of $10 \mathrm{~s}$ with the ultrasound probe on and off, and with the samples immersed in iced water to prevent heating.

\section{Differential scanning calorimetry}

The gel-liquid crystalline phase transitions of the DPPC liposomes with passively (molar ratios oleuropein:DPPC, from 1:10 to 2:1) and actively encapsulated oleuropein were determined by DSC (Nano DSC series III; Calorimetry Science, Provo, UT). DPPC-oleuropein systems samples were degassed and then loaded into the calorimetric cell, with 20 mM HEPES (pH 7.0) in the reference cell. Samples were then subjected to heating-cooling-heating in the temperature range of 15 to $70^{\circ} \mathrm{C}$, with heating/ cooling rates of $1{ }^{\circ} \mathrm{C} \mathrm{min}^{-}$

1 . The first DSC scans were used to determine the pretransition temperatures $\left(\mathrm{T}_{\text {pre }}\right)$, the gel-liquid phase transition temperatures $\left(\mathrm{T}_{\mathrm{m}}\right)$, and the model independent calorimetric enthalpies $\left(\Delta \mathrm{H}_{\text {cal }}\right)$. The thermodynamic parameters of the phase transitions of the lipids were determined from the DSC thermograms after baseline subtraction of the solvent (20 mM HEPES, pH 7.0), using the NanoAnalyze software (v3.10.0; Nano series Software; TA Instruments, New Castle, DE).

\section{Fluorescence emission polarization}

The fluorescent labels used were DPH and TMA-DPH, to follow the changes in membrane fluidity in the apolar inner part of the membrane and near to the membrane-water interface, respectively. The DPPC SUVs (at $1 \mathrm{mg} \mathrm{mL}^{-1}$ PL-90 g) without and with actively encapsulated oleuropein were diluted with $20 \mathrm{mM}$ HEPES ( $\mathrm{pH} 7.0$ ) to a final concentration of $0.1 \mathrm{mg} \mathrm{mL} \mathrm{m}^{-1}(136 \mu \mathrm{M})$. The DPH and TMA-DPH fluorophore stock solutions were then added to obtain a final concentration of $1 \mu \mathrm{M}$ and $2 \mu \mathrm{M}$, respectively. In the case of empty DPPC SUVs used for passive oleuropein encapsulation, the HEPES solution contained oleuropein to obtain a final concentration of $0 \mu \mathrm{M}$ (control, empty liposomes) to $272 \mu \mathrm{M}$, to provide molar ratios of oleuropein:DPPC $\left(n_{\text {Oleu }}\right.$ ' $n_{\text {DPPC }}$ ) of up to $2: 1$.

Fluorescence polarization was measured at an excitation wavelength of $358 \mathrm{~nm}$, with excitation polarizer in the vertical position, and the vertical and horizontal components of polarized light were recorded at $410 \mathrm{~nm}$. The data are expressed as change in fluorescence polarization $(\Delta P)$, as the effects of oleuropein addition compared to the control (no oleuropein addition). The ratio of the sensitiveness of detection for the vertically and horizontally polarized light, the G-factor, was determined separately for each sample before further measurements, in a 10-mm-path-length glass cuvette using a fluorescence spectrophotometer (Cary Eclipse; Varian, Australia) at 25 and $47{ }^{\circ} \mathrm{C}$, with band-pass filters of $5 \mathrm{~nm}$ for both excitation and emission.

\section{Lipid peroxidation kinetics}

To determine the kinetics of lipid peroxidation and the oleuropein antioxidant effects, a $2 \mathrm{mM}$ stock solution of the BODIPY 581/591 fluorescent probe was prepared in dimethylsulfoxide. This BODIPY stock $(1.25 \mu \mathrm{l})$ was mixed with $125 \mu \mathrm{l}$ PL-90 $\mathrm{g}$ SUVs (final concentration, $0.5 \mathrm{mg} \mathrm{mL}^{-1}$ ) in $2.35 \mathrm{~mL} 20 \mathrm{mM}$ HEPES (pH 7.0), and $25 \mu 1$ ethanol (no oleuropein control), or oleuropein in ethanol to have three 


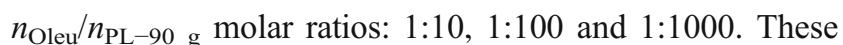
mixtures were incubated at $25{ }^{\circ} \mathrm{C}$ for 15 min under stirring inside quartz cuvettes in the dark, and peroxidation was induced by addition of $20 \mu 11 \mathrm{mM} \mathrm{CuCl}_{2}$ (final concentration, 8 $\mu \mathrm{M})$. For the background peroxidation control, $20 \mu \mathrm{l} 20 \mathrm{mM}$ HEPES (pH 7.0) was added instead. The kinetics of the oxidation reaction of BODIPY were monitored over $520 \mathrm{~min}$ using a fluorescence spectrophotometer (Cary Eclipse; Varian, Australia), with excitation wavelength of $500 \mathrm{~nm}$, and emission wavelength of $520 \mathrm{~nm}$.

\section{Thiobarbituric acid reacting substances}

Peroxidation of the PL-90 g liposomes with actively encapsulated oleuropein was determined using the thiobarbituric reacting substances (TBARS) assay. The PL-90 g liposomes (14 $\mathrm{mg} \mathrm{mL}^{-1}$ ) without (control) and with encapsulated oleuropein $\left(n_{\mathrm{Oleu}} / n_{\mathrm{PL}-90 \mathrm{~g}}\right.$ ratio, $\left.1: 3\right)$ were transferred to $3 \mathrm{~mL} \mathrm{UV-transparent} \mathrm{cuvettes} \mathrm{and} \mathrm{exposed} \mathrm{to} \mathrm{UV} \mathrm{radiation}$ at $254 \mathrm{~nm}$ and $50 \mathrm{~Hz}$ (Universal UV lamp; Camag, Muttenz, Switzerland) for $24 \mathrm{~h}$, positioned at a distance of $10 \mathrm{~cm}$. The nonirradiated control samples were kept in parallel in the dark. Aliquots of $0.2 \mathrm{~mL}$ were taken at specific times and mixed in screw-capped glass tubes with $3 \mathrm{~mL} \mathrm{20 \%}$ trichloroacetic acid and $1 \mathrm{~mL} 10 \%$ perchloric acid, $1 \%$ thiobarbituric acid. These samples were kept in a water bath at $100{ }^{\circ} \mathrm{C}$ for $25 \mathrm{~min}$. The reaction was quenched by cooling the samples in iced water for $5 \mathrm{~min}$, and the centrifuged at $1000 \times g$ for $8 \mathrm{~min}$. The pink colored compounds developed from the lipid hydroperoxides and the thiobarbituric acid reaction were analyzed by measurement of absorbance at $532 \mathrm{~nm}$ using a UV-Vis spectrophotometer (100 Bio Cary; Varian, Australia).

\section{pH stability of oleuropein in olive leaf extract solutions}

The stability of the compounds contained in the OLE was studied by following the degradation of its main biophenol constituent oleuropein at different pHs. An amount of OLE equivalent to $50 \mathrm{mg}$ oleuropein (i.e., $112.36 \mathrm{mg}$, at $44.5 \mathrm{wt} \%$ in OLE) was dissolved in $1 \mathrm{~mL}$ ethanol and then added to $40 \mathrm{~mL}$ of the following: $10 \mathrm{mM}$ citric acid/ $\mathrm{HCl}, \mathrm{pH} 2.0$;

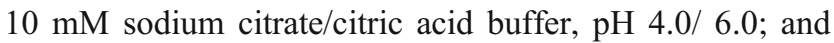
$10 \mathrm{mM}$ sodium phosphate buffer, $\mathrm{pH}$ 7.0. The resulting solutions were stirred for $30 \mathrm{~min}$ at $300 \mathrm{rpm}$, and then centrifuged to remove insoluble material $(5000 \times \mathrm{g}, 15 \mathrm{~min})$. The volume of each was then made up to $50 \mathrm{~mL}$ with the corresponding buffer (for final $1 \mathrm{mg} \mathrm{mL}^{-1}$ oleuropein). Finally, $10 \mathrm{~mL}$ of each of these OLE $\mathrm{pH}$ conditions were transferred to glass test tubes (in triplicate), which were incubated at $5{ }^{\circ} \mathrm{C}$ for 35 days. Samples were taken at different predefined times for determination of oleuropein concentrations, using HPLC.

\section{Liposome encapsulation of olive leaf extract}

The PL-90 g liposomes with encapsulated OLE ('OLE liposomes') were prepared as previously reported [16] using the proliposome method [23]. The OLE was most soluble in ethanol and oleuropein has an amphiphilic character, thus active encapsulation was performed as for lipophilic compounds by dissolving the OLE in the ethanol and phospholipids mixture. Here, $1 \mathrm{~g}$ PL-90 $\mathrm{g}$ was dissolved in $1 \mathrm{~g}$ ethanol and an amount of OLE that contained $50 \mathrm{mg}$ oleuropein was added to the mixture (112.36 $\mathrm{mg}$ at $44.5 \mathrm{wt} \%$ in OLE). Then $2 \mathrm{~mL}$ ultrapure water was added while stirring (magnetic bar), to form the phospholipid into bilayers. The mixture was heated to $60{ }^{\circ} \mathrm{C}$ for $2 \mathrm{~min}$ in a water bath, and then cooled to room temperature while adding $50 \mathrm{~mL}$ ultrapure water, drop-wise, and while stirring at $800 \mathrm{rpm}$, to allow formation of liposomes (with $\sim 1 \mathrm{mg} \mathrm{mL}^{-1}$ encapsulated oleuropein). Empty liposomes were prepared in the same way without the addition of OLE ('empty liposomes').

\section{Liposome size and zeta-potential}

Liposome size was measured by dynamic light scattering (Zetasizer Nano ZS; Malvern Instruments, Worcestershire, UK) at $25^{\circ} \mathrm{C}$, with a detector angle of $173^{\circ}$. The PL-90 g empty liposomes and OLE liposomes were conveniently diluted and transferred to a folded capillary cell (DTS1070). The mean size (i.e., hydrodynamic diameter) and the polydispersity index were obtained by photon correlation spectroscopy, with the data reported as intensity distributions. Zeta-potential was calculated from the electrophoretic mobility using the software for the Helmoltz-Smoluchowski model. Data are presented as means \pm standard deviation of five measurements.

\section{Liposome morphology}

The morphology and size of the empty and OLE liposomes were examined using transmission electron microscopy. Approximately $2 \mu 1$ of the samples were applied directly onto formvar-coated 400-mesh copper transmission electron microscopy grid for $30 \mathrm{~s}$. Before applying the samples, the grids were coated with carbon (approximately $4 \mathrm{~nm}$ ) and glow discharged at an intensity of $5 \mathrm{~mA}$ for $10 \mathrm{~s}$ (Leica EM ACE200). Staining was performed by application of a drop of $1 \%$ aqueous uranyl acetate onto the grid, which was drawn off the grid after a few seconds. The samples were examined under transmission electron microscopy (CM100; Philips) and electron micrographs were acquired using a digital camera (Orius 200; Gatan) and the Digital Micrograph software. These were further inspected and analyzed using the Image $\mathrm{J}$ software (Fiji). 


\section{Determination of biophenols encapsulation efficiency in OLE-liposomes}

The amounts of encapsulated oleuropein and verbascoside were determined by HPLC. The diluted liposomes were centrifuged $\left(12,000 \times \mathrm{g}, 180 \mathrm{~min}, 20^{\circ} \mathrm{C}\right)$, the supernatant was separated and analyzed, and the pelleted liposomes were resuspended in fresh ultrapure water. To determine the amounts of encapsulated compounds, these resuspended liposomes were disrupted by addition of $1 \mathrm{~mL}$ methanol and $1 \mathrm{~mL}$ chloroform. The mixture was vortexed thoroughly and left to allow phase separation. The concentrations of biophenols (oleuropein and verbascoside) in the upper water-methanol phase and in the supernatant were determined and considered as the encapsulated and nonencapsulated fractions, respectively. Encapsulation efficiency was calculated as in Eq. (1):

\footnotetext{
Encapsulation efficiency (\%)

$=$ mass of encapsulated biophenol $/$ total mass of biophenols $($ encapsulated + nonencapsulated $) \times 100$
}

\section{Oleuropein stability in and release from OLE liposomes}

Samples of OLE liposomes $(1.5 \mathrm{~mL})$ were diluted in aqueous buffer solutions $(10 \mathrm{~mL})$ at $\mathrm{pH} 2.0, \mathrm{pH} 4.0, \mathrm{pH} 6.0$ and $\mathrm{pH} 7.0$ (see above for details) and incubated at $5{ }^{\circ} \mathrm{C}$ for $500 \mathrm{~h}(\sim 21$ days). At predetermined times, aliquots were centrifuged $\left(12,000 \times g, 4 \mathrm{~h}, 20^{\circ} \mathrm{C}\right)$ to separate liposomes from the continuous phase. The OLE was extracted from liposomes as described in the previous section. The oleuropein concentrations were determined in both supernatant and liposome fractions, by HPLC.

\section{Incorporation of OLE and OLE liposomes into a commercial lemonade}

The OLE and the OLE liposomes ( $1 \mathrm{mg}$ oleuropein $\mathrm{mL}^{-1}$ ) were incorporated into two model 'drinks' and into commercial lemonade, to the final oleuropein concentration of $\sim 130$ $\mu \mathrm{g} \mathrm{mL} \mathrm{m}^{-1}$. The two model drinks were prepared to have the same $\mathrm{pH}$ without and with addition of the same vitamin C (Lascorbic acid) concentration as the commercial lemonade. These thus consisting of $10 \mathrm{mM}$ sodium citrate/citric acid buffer, $\mathrm{pH}$ 2.87, without and with $1.2 \mathrm{mg} \mathrm{mL}^{-1}$ ascorbic acid. All of these drink samples were incubated at $5{ }^{\circ} \mathrm{C}$ for 47 days, with analysis at predetermined times for the extraction of the OLE using methanol and chloroform (see previous sections), and determination of the total oleuropein content.

\section{Statistical analysis}

The data are expressed as means \pm standard deviation of $\mathrm{n}$ (as indicated) replicates. Analysis of variance (ANOVA) was used to compare the means by Tukey's multiple comparison tests $(p<0.05)$ using GraphPad Prism, version 8.1.1, for Mac (GraphPad Software, La Jolla, CA).

\section{Results and discussion}

\section{Differential scanning calorimetry}

Differential scanning calorimetry was used to measure the changes in the behavior of the thermotropic phase of the DPPC liposomes, and to determine the influence of oleuropein. Only membranes that consist of pure phospholipid with saturated fatty acids (i.e., DPPC) have cooperative (sharp) phase transitions that can be measured in a temperature range from 20 to $70{ }^{\circ} \mathrm{C}$. Thermograms of DPPC liposomes with increasing concentrations of passively encapsulated oleuropein (i.e., added after liposome formation) and actively encapsulated oleuropein (i.e., added during liposome formation) are shown in Fig. 1. The thermodynamic data for all of the samples are given in Table 1. These DPPC liposomes had the main endothermic lipid phase transition at $41.5^{\circ} \mathrm{C}$, which is known as the gel-to-liquid phase transition temperature $\left(\mathrm{T}_{\mathrm{m}}\right)$. They also showed a smaller and broader peak at around $36.5{ }^{\circ} \mathrm{C}$, which is known as the pretransition temperature $\left(\mathrm{T}_{\mathrm{m}}^{\prime}\right)$, whereby the membranes start to ripple and the gel and liquid lipid phases coexist [24].

The incorporation of oleuropein into liposome suspensions (i.e., passively encapsulation) at the lower oleuropein concentrations (Fig. 1a) did not significantly affect $\mathrm{T}^{\prime}{ }_{\mathrm{m}}$ or $\mathrm{T}_{\mathrm{m}}$. However, at the higher oleuropein concentrations, for the oleuropein:DPPC molar ratios $\geq 1.0$, there was a broadening and splitting of the peak for the $\mathrm{T}_{\mathrm{m}}$, and the pre-transition temperature peak nearly disappeared. A concentrationdependent decrease in the enthalpy of the pretransition from $5.5 \mathrm{~kJ} \mathrm{~mol}^{-1} \mathrm{~K}^{-1}$ to $\sim 1.0 \mathrm{~kJ} \mathrm{~mol}^{-1} \mathrm{~K}^{-1}$ was seen at oleuropein:DPPC molar ratios $\geq 1: 1$, while no significant trend was seen in the energy of the gel-to-liquid transition. Greater changes were seen when the oleuropein was actively encapsulated (Fig. 1b). The pretransition temperature peak $\mathrm{T}^{\prime}{ }_{\mathrm{m}}$ decreased by $3.1 \pm 0.2{ }^{\circ} \mathrm{C}$ and $\Delta H^{\prime}$ from $5.5 \pm 0.1$ to $1.5 \pm 0.2 \mathrm{~kJ} \mathrm{~mol}^{-1} \mathrm{~K}^{-1}$ at the 1:5 oleuropein:DPPC molar ratio, and then disappeared at the 1:1 molar ratio. 
Fig. 1 Differential scanning calorimetry thermograms (as measures of Cp: specific heat) of 1,2-dipalmitoyl-sn-glycero-3phosphocoline (DPPC) liposomes with passively (a) and actively (b) encapsulated oleuropein at different oleuropein:DPPC molar ratios (as indicated), in $20 \mathrm{mM}$ HEPES (pH 7.0)

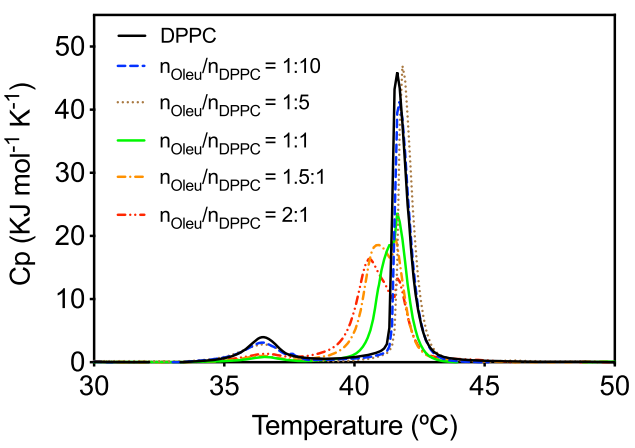

b

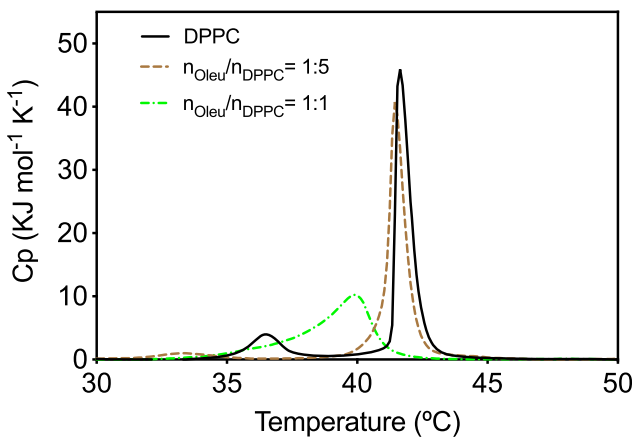

Concentration-dependent decreases were seen at $n_{\mathrm{Oleu}} / n_{\mathrm{DPPC}}$ of $1: 1$ for the main transition temperature $\mathrm{T}_{\mathrm{m}}$, by $1.7 \pm 0.1^{\circ} \mathrm{C}$, and $\Delta H$, by $5.2 \pm 0.5 \mathrm{~kJ} \mathrm{~mol}^{-1} \mathrm{~K}^{-1}$. This broadened or splitting of the main transition temperature peak with a shift to lower temperatures, and suppression of the pre-transition temperature peak, have been reported in DPPC liposomes with other biophenols, like resveratrol [25], and with flavonoids, like epicatechin and epigallocatechin [26]. Also, oleuropein showed similar thermotropic effects for the zwitterionic phospholipid dimyristoylphosphatidylcholine [18] and for the negatively charged phospholipids bilayers of dimyristoylphosphoglycerol [20]. These changes are associated with decreases in the transition cooperativity of the phospholipid acyl chains as a consequence of structural and dynamic changes in the membrane bilayers induced by oleuropein [27]. At higher concentrations, oleuropein can act as a spacer or 'interstitial impurity' while in a 'shallow' position within the membranes, through polar interactions with the phospholipid headgroups, to produce fluidifying effects without any large change in $\Delta H[18,26]$.
The more substantial changes in the liposomes with the actively encapsulated oleuropein might indicate that the oleuropein was also entrapped at a deeper membrane level and in the aqueous core, thus not only interacting at the level of the outer membrane surface.

\section{Effects of oleuropein on structural order and membrane fluidity}

Fluorescence polarization gives information about the degree of mobility of the molecular orientation within membranes while in the excited state. DPH is a non-polar fluorophore that can be used to track structural changes in the deeper regions of the phospholipid palisade of acyl chains. Conversely, TMADPH is an amphiphilic molecule that can be used to monitor changes closer to the polar headgroups of the membrane phospholipids [28]. DPH and TMA-DPH polarization increases (and thus their reorientation decreases) in the motionrestricted environment of the more ordered gel crystalline state.
Table 1 Thermodynamics data for the phase transitions of the 1,2-dipalmitoyl-sn-glycero-3phosphocoline (DPPC) liposomes at $\mathrm{pH} 7.0$ in the absence and presence of oleuropein according to encapsulation and molar ratio

\begin{tabular}{|c|c|c|c|c|}
\hline \multirow{2}{*}{$\begin{array}{l}\text { Oleuropein:DPPC } \\
\text { molar ratio }\end{array}$} & \multicolumn{2}{|l|}{ Pretransition } & \multicolumn{2}{|c|}{ Gel-to-liquid crystalline transition } \\
\hline & Temperature, $\mathrm{T}_{\mathrm{m}}\left({ }^{\circ} \mathrm{C}\right)$ & $\begin{array}{l}\text { Enthalpy, } \Delta H^{\prime} \\
\left(\mathrm{kJ} \mathrm{mol}^{-1} \mathrm{~K}^{-1}\right)\end{array}$ & Temperature, $\mathrm{T}_{\mathrm{m}}\left({ }^{\circ} \mathrm{C}\right)$ & 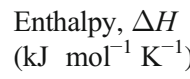 \\
\hline DPPC alone & $36.4 \pm 0.2$ & $5.5 \pm 0.1^{\mathrm{e}}$ & $41.6 \pm 0.1$ & $33.8 \pm 0.3^{\mathrm{d}}$ \\
\hline \multicolumn{5}{|c|}{ Passively encapsulated oleuropein } \\
\hline $1: 10$ & $36.2 \pm 0.2$ & $5.2 \pm 0.2^{\mathrm{e}}$ & $41.6 \pm 0.1$ & $29.7 \pm 0.1^{\mathrm{b}}$ \\
\hline $1: 5$ & $36.2 \pm 0.2$ & $4.5 \pm 0.1^{\mathrm{d}}$ & $41.5 \pm 0.1$ & $31.3 \pm 0.2^{\mathrm{c}}$ \\
\hline $1: 1$ & $36.4 \pm 0.2$ & $1.0 \pm 0.1^{\mathrm{b}}$ & $41.5 \pm 0.1$ & $28.0 \pm 0.5^{\mathrm{a}}$ \\
\hline $1.5: 1$ & $36.4 \pm 0.2$ & $1.1 \pm 0.1^{\mathrm{b}}$ & $41.5 \pm 0.1$ & $33.1 \pm 0.4^{\mathrm{d}}$ \\
\hline $2: 1$ & $36.4 \pm 0.2$ & $1.1 \pm 0.1^{\mathrm{b}}$ & $40.4 \pm 0.1^{\mathrm{a}}$ & $29.6 \pm 0.6^{\mathrm{b}}$ \\
\hline \multicolumn{5}{|c|}{ Actively encapsulated oleuropein } \\
\hline $1: 5$ & $33.3 \pm 0.2^{\mathrm{a}}$ & $1.5 \pm 0.2^{\mathrm{c}}$ & $41.4 \pm 0.1$ & $32.9 \pm 0.4^{\mathrm{d}}$ \\
\hline $1: 1$ & n.d. & n.d. & $39.9 \pm 0.1^{\mathrm{a}}$ & $28.6 \pm 0.5^{\mathrm{ab}}$ \\
\hline
\end{tabular}

Data are means \pm standard deviations $(n=02)$

Different superscript small letters in the same column indicate significant differences $(p<0.05$; pairwise difference with Tukey's test)

n.d., not detected 
Conversely, polarization is quenched when the lipid environment in which DPH and TMA-DPH are embedded is in the liquid disordered state, or when exposed to polar solvents.

The data for the fluorescence polarization changes $(\Delta P)$ of DPH and TMA-DPH in the DPPC liposomes with increasing concentrations of passively encapsulated oleuropein were initially investigated, at $25^{\circ} \mathrm{C}$ (Fig. 2a) and $47^{\circ} \mathrm{C}$ (Fig. 2b). Greater net fluorescent polarization was seen for both of these probes at $25^{\circ} \mathrm{C}$, at around $0.45-0.47$, which can be explained on the basis that at this temperature, DPPC is in the gel crystalline state, and is thus in a more ordered state. On the other hand, above the melting transition temperature, at $47^{\circ} \mathrm{C}$, the fluorescent polarization was lower (DPH, 0.12; TMA-DPH, 0.26 ). Addition of oleuropein to these liposome suspensions at $25{ }^{\circ} \mathrm{C}$ did not have any remarkable impact at either of the membrane levels, although at the highest molar ratios, as $\geq$ 1.5, there was a significant decrease in $\Delta P$ for TMA-DPH, which indicated a disordering effect at the outer membrane level. The lack of changes in the fluorescent polarization of $\mathrm{DPH}$ below $\mathrm{T}_{\mathrm{m}}$ (at $25^{\circ} \mathrm{C}$ ), when phospholipid hydrocarbon chains are in the gel crystalline state, suggested that oleuropein only interacts with the membrane phospholipids at a surface level, with interactions with the polar headgroups of DPPC, through constantly forming and breaking of $\mathrm{H}$-bonds. This correlates well with the DSC data here and with previous studies that used similar spin probes to assess the quenching of oleuropein fluorescence in dimyristoylphosphatidylcholine vesicles [20]. In another study, however, TMA-DPH fluorescent polarization did not significantly change in the presence of increasing oleuropein in soy phosphatidylcholine liposomes at $37^{\circ} \mathrm{C}$ [19]. However, it should be noted that at $37^{\circ} \mathrm{C}$, the phospholipids are already in the liquid fluid state, and thus small changes due to interactions with oleuropein might not have been detected.

When oleuropein was added during the liposome formation (i.e., actively encapsulated oleuropein), larger changes in polarization were seen. Figure 3 shows $\Delta P$ of the liposomes with

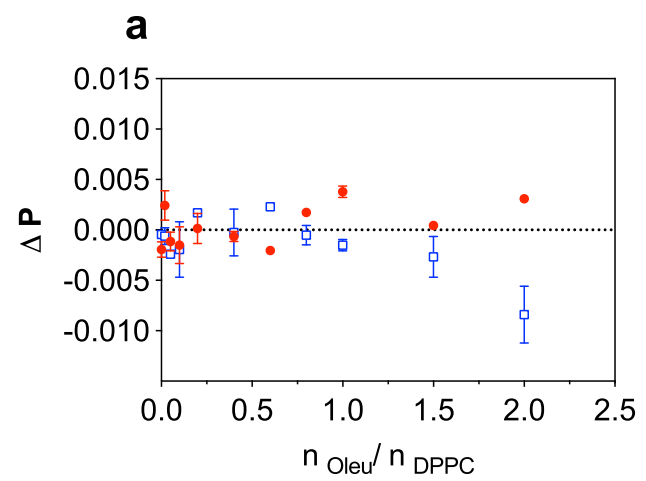

Fig. 2 Changes in polarisation $(\Delta \mathrm{P})$ of fluorophores 1,6-diphenyl-1,3,5hexatriene (DPH; •) and N,N,N-trimethyl-4-(6-phenyl-1,3,5-hexatrien-1yl) phenylammonnium p-toluenesulfonate (TMA-DPH; $\square$ ) added to 1,2dipalmitoyl-sn-glycero-3-phosphocoline (DPPC) small unilamellar this actively encapsulated oleuropein in more direct comparisons with the passively encapsulated oleuropein. The larger changes in $\Delta P$ for both DPH and TMA-DPH probes for the active encapsulation compared with the passive encapsulation indicated greater insertion of oleuropein into, and interaction within, the phospholipid multilamellae. When the phospholipid palisade is in the crystalline state (Fig. 3a, b), at the lower oleuropein concentration $\left(n_{\mathrm{Oleu}} / n_{\mathrm{DPPC}}=0.2\right)$, both of these probes showed small increases in fluorescence polarization, although much larger decreases were seen at the higher oleuropein concentrations. Again, this might indicate the positioning of the oleuropein more towards the surface level, as seen by the larger drop in fluorescence polarization, but also toward deeper levels closer to the acyl chains, as a consequence of oleuropein entrapment within the bilayers and lamellae during this active encapsulation liposome formation.

On the other hand, above the gel-to-liquid transition temperature (at $47{ }^{\circ} \mathrm{C}$ ), the liposomes with actively encapsulated oleuropein showed a greater significant increase in $\Delta P$ compared with those with passively encapsulated oleuropein (Fig. 3c, d), especially for DPH. This indicated that the actively encapsulated oleuropein was inserted in the membranes at different depths, and also that there were larger amounts of interacting or encapsulated oleuropein compared with its passive encapsulation. The increases in DPH and TMA-DPH fluorescent polarization showed that the membranes were less fluid (i.e., had a higher degree of ordering) following active encapsulation of oleuropein, and that this oleuropein was also at deeper levels in the liquid-state multilamellae, to produce a 'packing' effect. This behavior has been reported previously for other phenolic compounds, like resveratrol [25] rosemary polyphenols [29] and cholesterol and plant sterols [30].

\section{Lipid oxidation kinetics}

Lipid peroxidation represents the major degradation process in lipid systems like liposome membranes, and is thus a

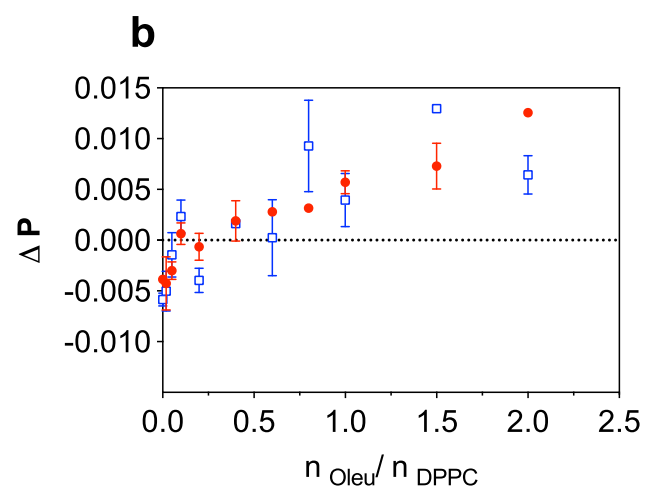

vesicles with passively encapsulated oleuropein at different molar ratios of oleuropein to DPPC ( $n_{\text {Oleu }} / n_{\text {DPPC }}$ ), analysed at 25 (a) and 47 (b). Data are means \pm standard deviation $(\mathrm{n}=02)$ 
Fig. 3 Changes in polarisation $(\Delta \mathrm{P})$ of fluorophores $1,6-$ diphenyl-1,3,5-hexatriene (DPH; a, c) and N,N,N-trimethyl-4-(6phenyl-1,3,5-hexatrien-1-yl) phenylammonnium ptoluenesulfonate (TMA-DPH; $\mathbf{b}$, d) added to 1,2-dipalmitoyl-snglycero-3-phosphocoline (DPPC) small unilamellar vesicles with passively (PE; $\square$ ) and actively $(\mathrm{AE} ; \mathbf{-})$ ecapsulated oleuropein at $25^{\circ} \mathrm{C}(\mathbf{a}, \mathbf{b})$ and $47^{\circ} \mathrm{C}(\mathbf{c}, \mathbf{d})$ and at different molar ratios of oleuropein to DPPC ( $n_{\text {Oleu }}$ ' $n_{\text {DPPC }}$. Data are means \pm standard deviation $(\mathrm{n}=02)$
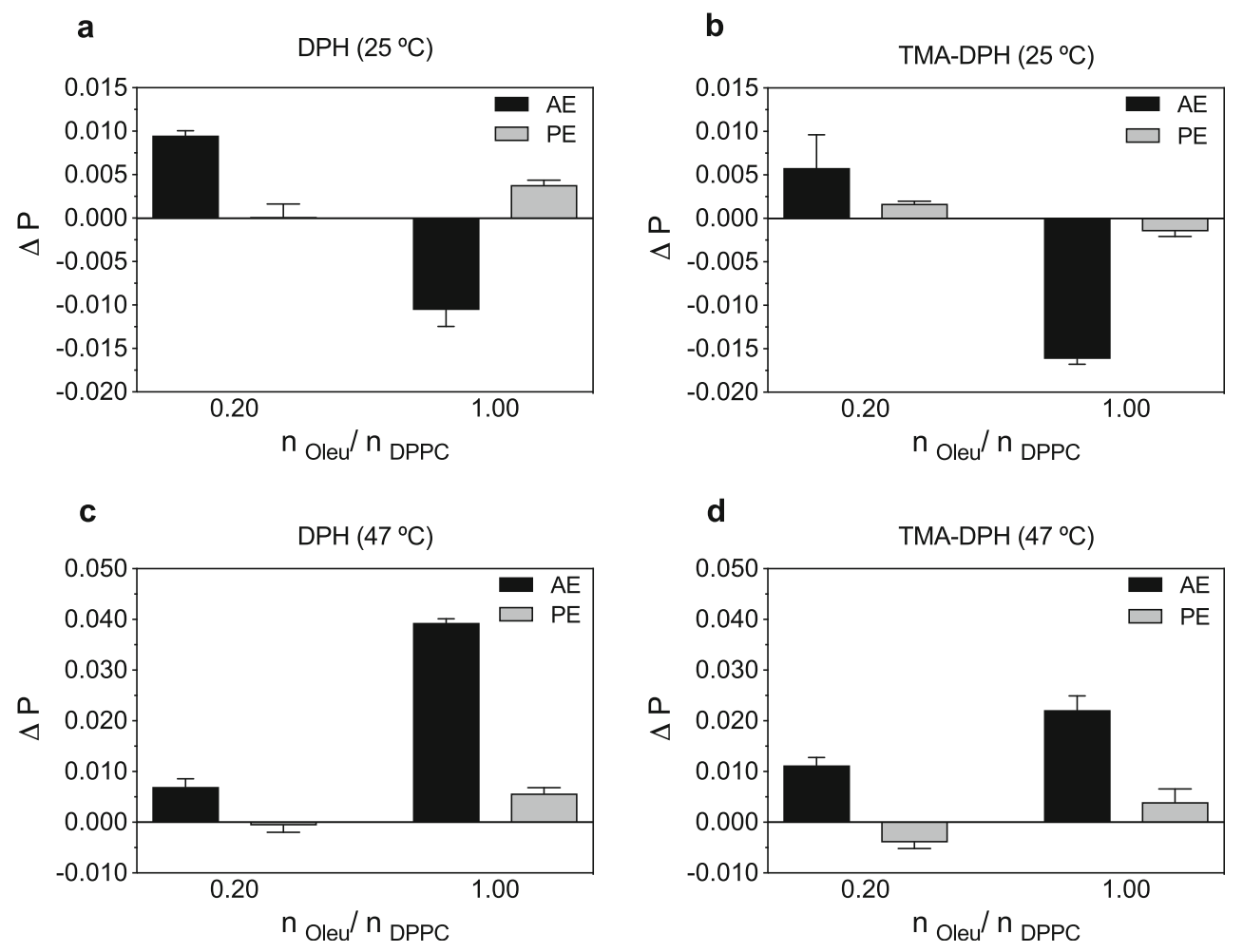

limiting factor for long-term stability and shelf-life. This process involves the unsaturated fatty acids in phospholipids, which represent susceptible targets for free radicals [31] BODIPY 581/591 $\mathrm{C}_{11}$ can be used as a lipid peroxidation marker to determine the antioxidant activities of different compounds in lipid membranes, as it can accommodate within membranes, and its fluorescence emission shifts to shorter wavelengths upon oxidation [32].

The antioxidant effects of actively encapsulated oleuropein in SUVs prepared with PL-90 $\mathrm{g}$ was tested in a dose- and timedependent assay of BODIPY 581/591 $\mathrm{C}_{11}$ oxidation kinetics. Figure 4 shows the oxidation kinetics in the absence and presence of encapsulated oleuropein at three different oleuropein:PL-90 g molar ratios, and for the control, to allow for the intrinsic level of peroxidation (i.e., in the absence of the added oxidant, $\mathrm{CuCl}_{2}$ ). The insert in Fig. 4 shows more detail for the differences between the oxidation kinetics in the initial stages. As can be noted, all of the samples except the control showed increases in BODIPY 581/591 fluorescence intensity over time. However, the differences in the oxidation kinetics and the final net oxidation at 520 min changed as a function of the oleuropein concentration. At the highest oleuropein concentration $\left(n_{\mathrm{Oleu}} / n_{\mathrm{PL}-90 \mathrm{~g}}=1: 10\right)$, lipid oxidation was almost completely inhibited $(95.8 \% \pm 0.01 \%)$, with no significant difference from the nonoxidized control $(p=0.205)$. At the intermediate concentration $\left(n_{\mathrm{Oleu}} / n_{\mathrm{PL}-90 \mathrm{~g}}=1: 100\right)$, oleuropein significantly reduced oxidation $(61.2 \% \pm 0.2 \%)$, while with oleuropein at one order of magnitude lower $\left(n_{\mathrm{Oleu}} / n_{\mathrm{PL}-90 \mathrm{~g}}=\right.$
$1: 1000)$, there remained low but significant inhibition of $(5.8 \% \pm 0.2 \% ; p=0.031)$ compared to the liposomes without oleuropein encapsulation $\left(n_{\mathrm{Oleu}} / n_{\mathrm{PL}-90 \mathrm{~g}}=0\right)$. Therefore, oleuropein had a protective effect on the lipid peroxidation.

The mechanism of the antioxidant effects of such phenolic compounds has generally been attributed to the breaking of the radical oxidation propagation of peroxyradicals ( $\mathrm{LOO}^{\circ}$ ) generated during the initiation of this process by external agents, such as with ionic radiation or photon impact, or as

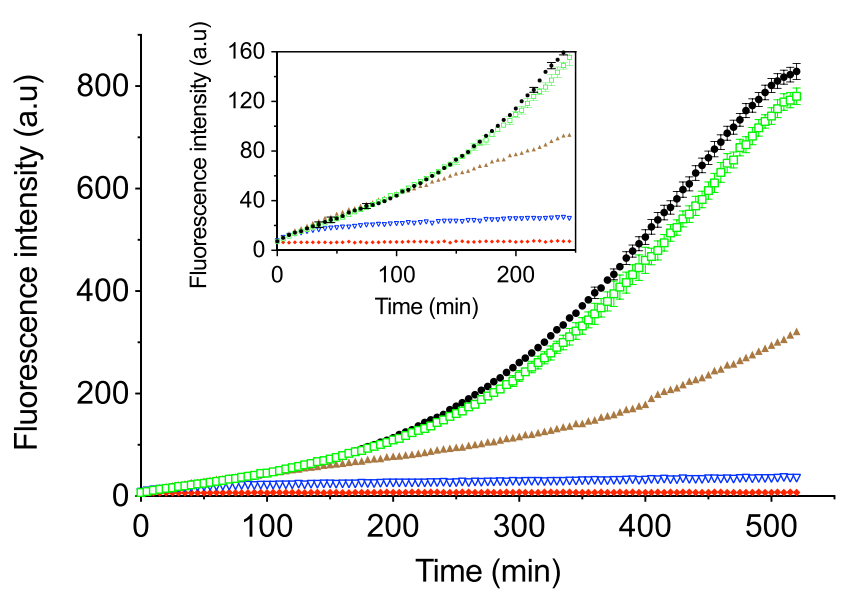

Fig. 4 BODIPY 581/591 $\mathrm{C}_{11}$ oxidation kinetics in PL-90 g small unilamellar vesicles prepared with actively encapsulated oleuropein at different molar ratios of oleuropein to PL-90 g $\left(n_{\text {Oleu }} / n_{\mathrm{PL}-90 \mathrm{~g}}\right)$ : $\bullet$, no oleuropein control; $\square, 1: 1000 ; \wedge, 1: 100 ; \nabla, 1: 10 ; \diamond$, no oxidant control, at $25^{\circ} \mathrm{C}$ 
in the present analysis, metal ions [33]. Phenolic compounds donate a hydrogen radical to $\mathrm{LOO}^{\circ}$, and hence they can delay the propagation of lipid oxidation across the membranes. However, previous studies have shown that the protective effects of phenolics might also arise from their increased membrane bilayer ordering and packing. This will promote steric hindrance for radical propagation, and will decrease the acyl chain hydration, as has been shown for cholesterol and phenolics [29, 30, 34]. A study by Balanč [25] with resveratrol showed similar but more pronounced effects on the thermotropic properties and packing of DPPC membranes compared to the present study. Also, the inhibition of BODIPY 581/591 oxidation by resveratrol was higher, which supports synergistic effects of physical stabilization and radical scavenging mechanisms.

\section{Thiobarbituric acid reacting substances}

To investigate the antioxidant effects of oleuropein here, liposome peroxidation was induced using UV-C irradiation. This approach measures the extent of malondialdehyde formation, a decomposition product of lipid hydroperoxides. UV radiation can trigger a peroxidation initiation reaction in the fattyacid double bonds, to thus promote the oxidation cascade [33].

The PL-90 g liposomes without (control) and with actively encapsulated oleuropein $\left(n_{\mathrm{Oleu}} / n_{\mathrm{PL}-90 \mathrm{~g}}=1: 3\right)$ were treated with UV light and compared with the same samples stored in the dark. The data for the TBARS levels as a measure of peroxidation of these PL-90 g liposomes are presented in Fig. 5. None of the samples stored in the dark showed any

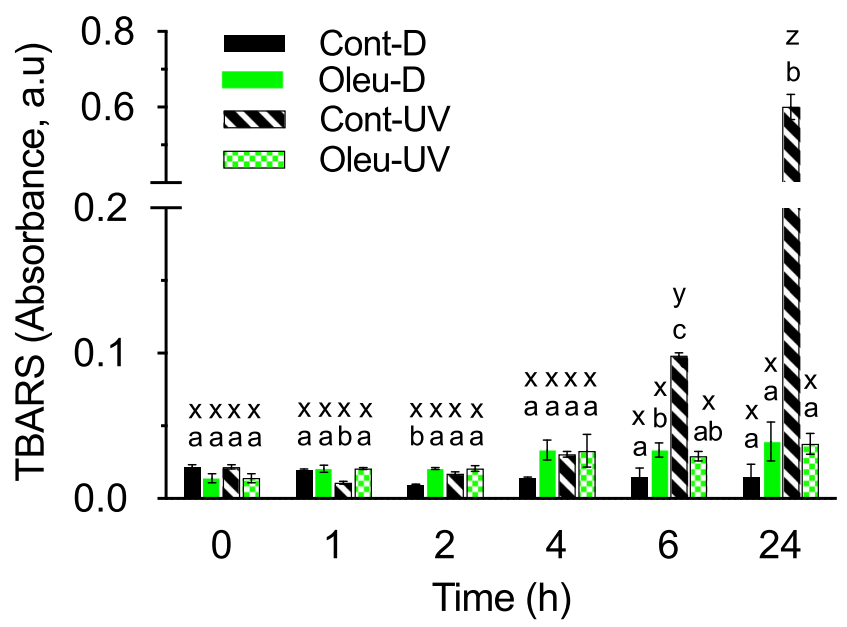

Fig. 5 Peroxidation of PL-90 g liposomes with actively encapsulated oleuropein (Oleu) using the thiobarbituric reacting substances (TBARS) assay following treatment with UV light (UV) and in the dark (D) at $25^{\circ} \mathrm{C}$. Data are means \pm standard deviation $(n \geq 02)$. Data with different letters a to $\mathrm{c}$ within each time point or with different letters $\mathrm{x}$ to $\mathrm{z}$ under the same conditions across the time points are significantly different $(p<0.05)$ particular changes in TBARS over the time tested. For the control samples exposed to UV light (Fig. 5, Cont-UV), the TBARS levels started to increase after $4 \mathrm{~h}$, to reach 5 -fold and 30 -fold the TBARS levels after 6 and $24 \mathrm{~h}$, respectively. However, with actively encapsulated oleuropein in the PL$90 \mathrm{~g}$ liposomes, this lipid peroxidation was almost completely inhibited after the $24 \mathrm{~h}$ treatment, with only a 3 -fold increase in the TBARS levels seen $(p=0.046)$; also, no significant differences were seen in the TBARS levels for the PL-90 $\mathrm{g}$ liposomes stored in the dark. This thus demonstrated the antioxidant potential of oleuropein in these PL-90 $\mathrm{g}$ liposomes.

As already discussed, oleuropein is believed to have two mechanisms involved in its antioxidant effects: scavenging of the $\mathrm{O}_{2}$ radicals generated by UV light, and a biophysical effect through an increase in membrane ordering and steric hindrance of $\mathrm{LOO}^{\circ}$ propagation. The amphiphilic nature of oleuropein and its surface location within the membranes means that it can scavenge the free radicals in the aqueous medium. During the oleuropein encapsulation, some of the oleuropein might also become positioned in the internal membrane lamellae and at deeper levels in the lipid bilayer, as observed using DSC and fluorescence polarization. PaivaMartins et al. [19] reported that oleuropein and other olive biophenols have greater antioxidant activities than tocopherols and Trolox against 2,2'-azobis(2-amidinopropane) dihydrochloride-induced peroxidation, because of an increased delay in the lag phase of oxidation. This possibility to delay peroxidation initiation was associated with their effectiveness as radical scavengers in the aqueous environment in the vicinity of the phospholipid bilayers. However, they also observed that olive biophenols are not as good for scavenging of lipid peroxyl radicals during the propagation stage, which might also explain the long lag phase observed in the present study, and the subsequent large increase after $6 \mathrm{~h}$ of $\mathrm{UV}$ irradiation. They also showed that $\alpha$-tocopherol, which is a more hydrophobic molecule that sits in the core of the membranes, was more effective for slowing of the peroxidation rate due to its scavenging of lipid peroxyl radicals. This indicates that it might be better to use the synergistic effects of hydrophobic and amphiphilic/ hydrophilic antioxidants when formulating liposome suspensions.

\section{HPLC determination of main phenolic compounds in the OLE}

The HPLC analysis of the phenolic composition of the OLE showed that oleuropein was the main component (Online Resource 1, Fig. 1, peak 3), and that it accounted for nearly half of the dry mass, as $445 \pm 5 \mathrm{mg}$ oleuropein $\mathrm{g}^{-1}$ OLE. The other phenolics in particular in this analysis were verbascoside $\left(22.9 \pm 0.2 \mathrm{mg} \mathrm{g}^{-1}\right.$ OLE) and hydroxytyrosol $(3.9 \pm 0.1 \mathrm{mg}$ $\mathrm{g}^{-1}$ OLE). 


\section{Encapsulation efficiency of verbascoside and oleuropein in the OLE liposomes}

The encapsulation efficiencies for the two main biophenols in the OLE, as oleuropein and verbascoside, were determined for OLE liposomes prepared with a final concentration in the suspension of $\sim 1 \mathrm{mg} \mathrm{mL}^{-1}$ oleuropein. The encapsulation efficiency of oleuropein was $33.8 \% \pm 1.5 \%$, while that of verbascoside was higher, at $75.0 \% \pm 0.8 \%$. These encapsulation efficiencies of oleuropein were similar to those seen for other studies that investigated the pure compounds and olive extracts. Liposome that were prepared using the thin-film method for active encapsulation of oleuropein and hydroxytyrosol with two types of phospholipids showed encapsulation efficiencies of $\sim 30 \%$ for oleuropein and $\sim 12 \%$ for hydroxytyrosol [35]. Trucillo et al. [36] showed that encapsulation of the total phenolics from olive pomace ranged from $25-55 \%$ depending on their process parameters and the phospholipid:total phenolics ratio. Also, encapsulation of hydroxytyrosol in thin-film liposomes was reported to reach $40-45 \%$ [37], although a higher efficiency of $70-80 \%$ for total phenolics was reported for nanoliposomes prepared using the ethanol injection technique [38]. With respect to verbascoside, such particularly high encapsulation despite being a relatively polar molecule (similar to oleuropein) has been shown previously [39]. In this case, the entrapment efficiency for verbascoside was from $57-66 \%$ in liposomes made from soy phosphatidylcholine using the thinfilm method. Another study has also reported a deep location for verbascoside in phosphatidylcholine membranes [40], and another showed that it can provide unusually high inhibition of lipid peroxidation in liposomes [41]. It should also be noted here that the verbascoside content in relation to the phospholipids was considerably lower than that for oleuropein, which might go part way towards explaining its higher encapsulation efficiency. Indeed, in some of our earlier exploratory trials in which OLE liposomes were prepared with a final oleuropein concentration of $\sim 0.5 \mathrm{mg} \mathrm{mL}^{-1}$, the encapsulation efficiency was $\sim 42 \%$.

\section{Liposome size, zeta-potential and morphology}

The hydrodynamic size distributions were measured for empty liposomes and OLE liposomes using dynamic light scattering, as shown in Online Resource 1, Fig. 2. It can be noted here that empty liposomes and OLE liposomes were similar in size, with a range from $100 \mathrm{~nm}$ to $1,000 \mathrm{~nm}$ in diameter. However, compared to the mean diameter of empty liposomes (344 $\pm 6 \mathrm{~nm})$, OLE liposomes were larger $(405 \pm 4 \mathrm{~nm})$, and showed a higher polydispersity index $(0.31$ vs.. 0.35 , respectively) (Table 2). A small fraction of larger particles, at around $3 \mu \mathrm{m}$ to $5 \mu \mathrm{m}$, were also seen here, and these might be attributed to small aggregates of liposomes. Other studies using the same liposome preparation procedure to encapsulate resveratrol [15] and pantothenic acid [42] have reported liposomes with similar size ranges, from around $100 \mathrm{~nm}$ to $400 \mathrm{~nm}$ in diameter.

For the zeta potentials, those for liposomes and OLE liposomes under the different buffer pHs are given in Table 2 . Both of these liposome preparations showed negative charges in water suspension, and the OLE addition promoted small but significant increases the zeta potentials up to $\mathrm{pH} 6.0$, with a small, but significant, decrease at $\mathrm{pH}$ 7.0. At $\mathrm{pH} 2.0$, both liposome preparations showed high positive zeta potentials, while at $\mathrm{pHs}$ of 4.0 and 6.0 , they were close to 0 , with a returned to mildly negative at $\mathrm{pH}$ 7.0. The zeta potential is a measure of the extent of particle interactions and stabilities. Despite some literature inconsistencies, in general, the greater the surface charge, the greater the particle repulsion, and consequently the greater the physical stability [43]. Indeed, these liposome preparations at $\mathrm{pHs} 4.0$ and 6.0 showed incipient aggregation and sedimentation over time. On the other hand, at $\mathrm{pH} 2.0$ and in water, these liposomes showed excellent stability over long periods of storage at refrigeration temperatures (i.e., to $500 \mathrm{~h}$ ). Liposomes prepared with phosphatidylcholine typically have a net negative charge [15, 44]. Along with other phenolic compounds, the OLE constituents, and in particular oleuropein, can interact with the phosphate groups and glycerol in the polar headgroups of the phospholipids through hydrogen bonding and electrostatic interactions [14]. This results in changes to the surface potential and curvature of the membranes, with different patterns of phospholipid conformations, which explains also the differences in size $[38,45]$.

Representative electron microscopy images of empty liposomes and OLE liposomes are shown in Fig. 6. Overall, all of these liposomes showed regular round shapes, although some irregular and amorphous vesicles were also seen, which was more frequent for these empty liposomes (Fig. 6a). OLE liposomes appeared to have slightly larger sizes, as seen by the zsize analysis. Characteristic structures were also seen on the surfaces of OLE liposomes, which appeared to be either small vesicles or twisted lamellae. This might be due to effects of the OLE constituents on the membrane potential and curvature, which has also been reported previously in oleuropeinphospholipid interaction studies [20].

\section{Oleuropein stability and release from OLE liposomes}

The pH stability of oleuropein was determined in free OLE solutions at refrigeration temperatures $\left(5^{\circ} \mathrm{C}\right.$ ) (Online Resource 1, Fig. 3). It can be seen that the rate of degradation of the oleuropein in the OLE was higher at $\mathrm{pHs} 2.0$ and 7.0, with $74.8 \% \pm 1.2 \%$ and $77.5 \% \pm 2.0 \%$, respectively, remaining after 21 days of storage, and $62.3 \% \pm 0.6 \%$ and $67.1 \% \pm 0.3 \%$ after 35 days of storage. On the other hand, at pHs 4.0 and 6.0, these OLE solutions did not show significant oleuropein 
Table 2 Hydrodynamic and zeta potential measures using dynamic light scattering and electrophoretic mobility, respectively, for empty PL-90 g liposomes and PL-90 g liposomes with OLE (OLE liposomes) in water and $10 \mathrm{mM}$ buffers at indicated pHs at $25^{\circ} \mathrm{C}$

\begin{tabular}{|c|c|c|c|c|c|c|c|}
\hline \multirow[t]{3}{*}{ Liposome } & \multicolumn{2}{|c|}{ Hydrodynamic diameter } & \multicolumn{5}{|c|}{$\mathrm{pH}$ dependency of zeta potential (mV) } \\
\hline & \multirow{2}{*}{$\begin{array}{l}\text { Size } \\
(\mathrm{nm})\end{array}$} & \multirow{2}{*}{$\begin{array}{l}\text { Polydispersity } \\
\text { index }\end{array}$} & \multirow[t]{2}{*}{ Water } & \multicolumn{4}{|l|}{ Buffer $\mathrm{pH}$} \\
\hline & & & & 2.0 & 4.0 & 6.0 & 7.0 \\
\hline Empty & $344 \pm 6^{\mathrm{a}}$ & $0.31 \pm 0.04$ & $-49.2 \pm 1.0^{\mathrm{aA}}$ & $26.6 \pm 2.9^{\mathrm{aB}}$ & $-0.8 \pm 0.2^{\mathrm{aC}}$ & $-4.2 \pm 1.2^{\mathrm{aD}}$ & $-6.2 \pm 0.3^{\mathrm{aD}}$ \\
\hline OLE & $405 \pm 4^{\mathrm{b}}$ & $0.35 \pm 0.03$ & $-24.8 \pm 0.3^{\mathrm{bA}}$ & $31.5 \pm 1.5^{\mathrm{bB}}$ & $0.5 \pm 0.2^{\mathrm{bC}}$ & $-2.5 \pm 0.8^{\mathrm{bD}}$ & $-7.3 \pm 0.3^{\mathrm{bE}}$ \\
\hline
\end{tabular}

Data are means \pm standard deviation $(\mathrm{n}=03)$

Different superscript small (same column) and capital (same row) letters indicate significant differences ( $\mathrm{p}<0.05$; pairwise difference Tukey's test) $\mathrm{pH}$ 2.0, $10 \mathrm{mM}$ citric acid/HCl; $\mathrm{pH}$ 4.0/6.0, $10 \mathrm{mM}$ sodium citrate/citric acid buffer ; $\mathrm{pH} 7.0,10$ mM sodium phosphate buffer

degradation, with $98.2 \% \pm 1.0 \%$ and $92.3 \% \pm 3.0 \%$ remaining after 35 days of storage. Therefore, this degradation of oleuropein in the OLE is $\mathrm{pH}$ dependent, with high stability at $\mathrm{pHs}$ from 4.0 to 6.0., and significant lability at low $(\mathrm{pH} 2.0)$ and neutral ( $\mathrm{pH} 7.0) \mathrm{pHs}$. Oleuropein hydrolysis at acidic and neutral to alkaline $\mathrm{pH}$ has been reported previously, especially in studies on the debittering of olives, and the products that result from this oleuropein hydrolysis are hydroxytyrosol and elenolic acid [10, 46, 47]. Indeed, in the present study there was a steady linear increase on hydroxytyrosol concentrations in the OLE solutions at $\mathrm{pH} 2.0$ (data not shown).

The oleuropein stability and release from OLE liposomes was determined by measurements of the oleuropein concentrations in the liposome fractions (as encapsulated) and in the continuous aqueous phases (as nonencapsulated) over $500 \mathrm{~h}$ ( $\sim 21$ days) at $5{ }^{\circ} \mathrm{C}$ (Fig. 7). The greatest changes here were for OLE liposomes at $\mathrm{pH} 2.0$ (Fig. 7a). The nonencapsulated fraction of oleuropein, which accounted for $\sim 66 \%$ of the total oleuropein in OLE liposomes (thus with $\sim 34 \%$ encapsulated), was rapidly degraded, for a $60.4 \%$ loss after $44 \mathrm{~h}$ of storage (start, $98.4 \pm 0.8 \mu \mathrm{g} \mathrm{mL}^{-1}$; $44 \mathrm{~h}, 39.0 \pm 1.2 \mu \mathrm{g} \mathrm{mL}^{-1}$ ), which rose further to an $86.6 \%$ loss after $92 \mathrm{~h}\left(13.1 \pm 0.1 \mu \mathrm{g} \mathrm{mL} L^{-1}\right)$. On the other hand, the encapsulated fraction showed greater stability, with $70.5 \% \pm 2.3 \%$ remaining after $92 \mathrm{~h}$ of storage, and $48.2 \% \pm 1.5 \%$ remaining after $500 \mathrm{~h}$ of storage (Fig. $7 \mathrm{a}$, inset). This shows the stabilizing effect of this liposome encapsulation against oleuropein hydrolysis under these highly acidic conditions. In a similar study, liposome-encapsulated hydroxytyrosol showed greater stability than at $\mathrm{pH} 7.4$ over 30 days of storage at 4 and $25^{\circ} \mathrm{C}$ [37]. Liposome stabilizing effects have also been seen for other polyphenols, like epigallocatechin gallate [16]. On the assumption that these decreases in the concentrations of the encapsulated fraction of oleuropein are not associated with its hydrolysis, but are instead only due to its release/ leakage from the liposomes, the higher release rate at $\mathrm{pH} 2.0$ compared to higher $\mathrm{pHs}$ can be attributed to the lower stability of these liposomes under acidic conditions [48]. This effect has also been reported in previous studies of encapsulated bioactives in phosphatidylcholine liposomes $[16,42]$.

On the other hand, OLE liposomes at the other pHs showed different profiles. The total oleuropein content here remained
Fig. 6 Representative transmission electron micrographs of PL-90 g liposomes without (a) and with (b) encapsulated OLE a

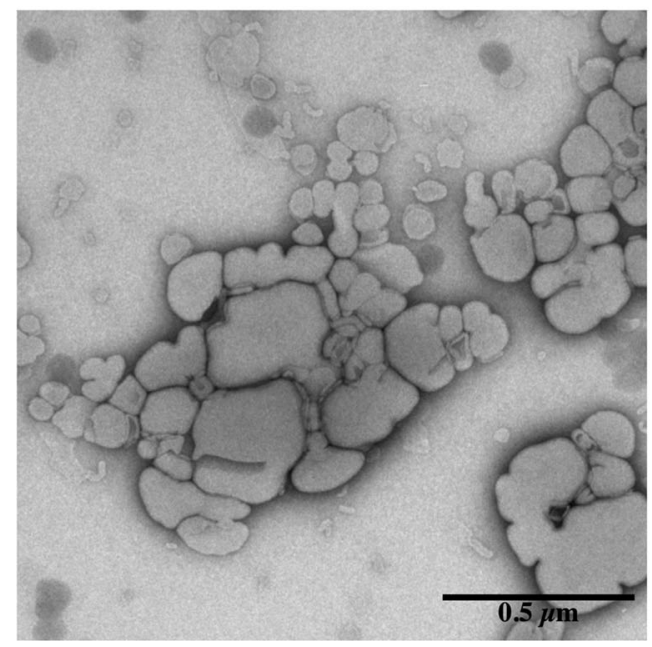

b

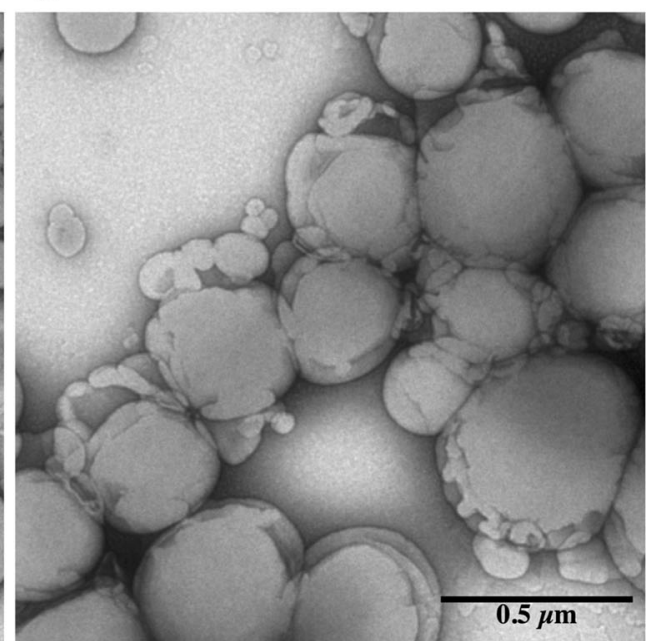


Fig. 7 Time courses of oleuropein stability and release from PL-90 g OLE liposomes in $10 \mathrm{mM}$ citric acid/HCl pH 2.0 (a), $10 \mathrm{mM}$ sodium citrate/citric acid buffer $\mathrm{pH} 4.0$ (b) and $\mathrm{pH} 6.0$ (c), and $10 \mathrm{mM}$ sodium phosphate buffer $\mathrm{pH} 7.0(\mathbf{d})$, at 5 . Data represent nonencapsulated (supernatant) ( $\square$ ) and encapsulated $(\bullet)$ oleuropein, as means \pm standard deviation $(\mathrm{n}=03)$. (a) inset, same data as (a) expressed as proportions of oleuropein remaining in the nonencapsulated and encapsulated fractions, in the PL-90 g OLE liposomes at pH 2.0
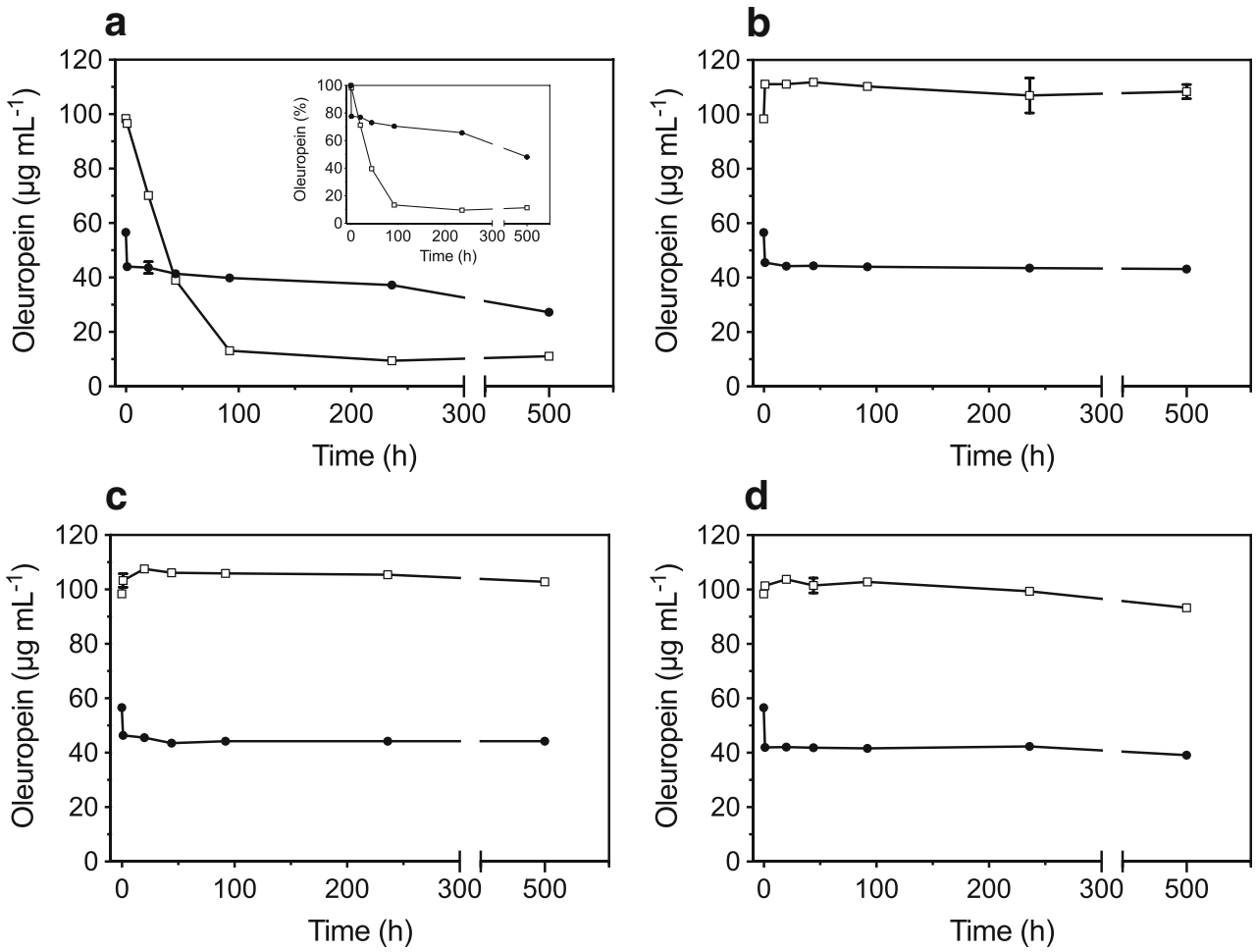

stable at pHs 4.0 and 6.0, with little or no degradation seen for both fractions. There was an initial release of $\sim 10 \mathrm{mg} \mathrm{mL}^{-1}$ oleuropein over the first hour (about $20 \%$ of its initial encapsulation), which was followed by a plateau with little or no release over the rest of the $500 \mathrm{~h}$ of storage. At $\mathrm{pH} 7.0$, the nonencapsulated fraction of oleuropein also decreased during storage as a consequence of oleuropein degradation.

This initial release of about $20 \%$ of the oleuropein from the encapsulated fraction might be associated with oleuropein that has weak surface interactions with the phospholipid membranes. Conversely, the negligible release over the rest of this storage period at $5{ }^{\circ} \mathrm{C}$ indicates that the liposome membranes were stable and maintained the oleuropein trapped inside. Under refrigeration conditions, PL-90 g liposomes undergo liquid-to-gel phase transition $\left(25-30^{\circ} \mathrm{C}\right)$ [49], which makes them more resistant to leakage and disruption as a result of their lower membrane fluidity $[50,51]$.

\section{Incorporation of OLE and OLE liposomes and oleuropein stability in a commercial lemonade drink}

To test the performance of OLE and OLE liposomes in a food matrix, a lemonade drink and two model drinks were enriched with both free OLE and PL-90 g liposome encapsulated OLE, with the total oleuropein content monitored over 47 days.

The protection of the oleuropein over time is shown in Fig. 8 for each of these three drink systems, and for the addition of both free and liposome-encapsulated OLE. Oleuropein
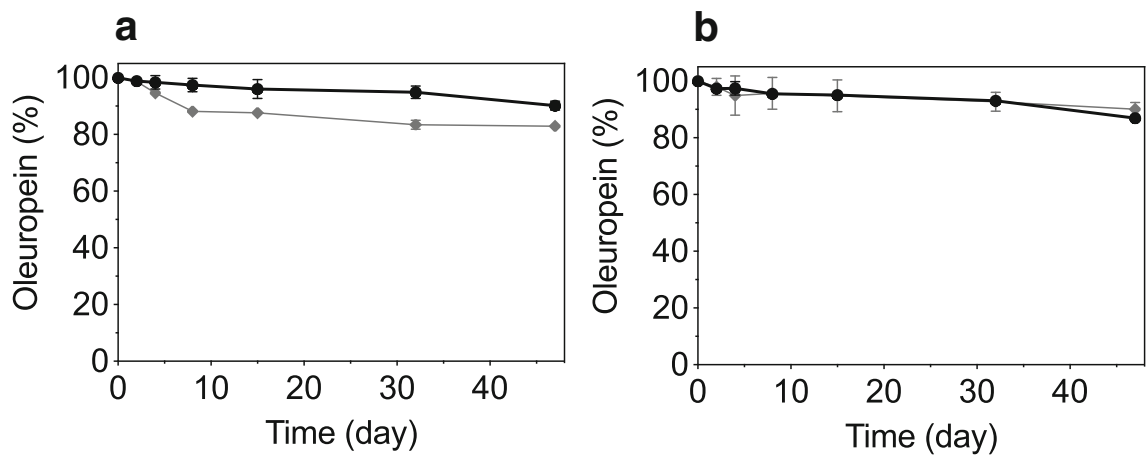

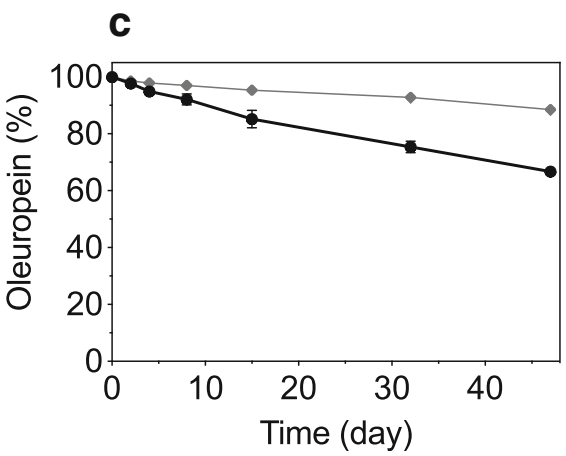

Fig. 8 Time courses of oleuropein stability when added as free OLE (•) or PL-90 g OLE liposomes $(\diamond)$ to a commercial lemonade drink (a), and to a 'model drink' of $10 \mathrm{mM}$ sodium citrate/citric acid buffer, $\mathrm{pH} 2.87$, with $1.2 \mathrm{mg} \mathrm{mL}^{-1}$ ascorbic acid (b) and without the ascorbic acid (c), at 5 ${ }^{\circ} \mathrm{C}$. Data are means \pm standard deviation $(n=03)$ 
showed good stability in the commercial lemonade drink (Fig. 8a) in both its free and encapsulated forms, with $90 \%$ and $83 \%$ remaining, respectively, after these 47 days. Perhaps surprisingly, the oleuropein was significantly more stable $(p<0.05)$ with the addition of free OLE compared to OLE liposomes. In the model system with ascorbic acid, there were no significant differences seen, and the oleuropein was particularly stable over the 47 days of storage, with only 10-13\% loss. However, in the simpler system without ascorbic acid, the differences were more substantial, and OLE liposomes appeared to have a protective effect on oleuropein stability. The oleuropein concentration in this system with free OLE decreased steadily over the 47 days, with $67 \%$ remaining after 47 days, while for OLE liposomes, more oleuropein remained after the 47 days (88\%). It appears that ascorbic acid can delay acid hydrolysis of the oleuropein, as seen by its lower degradation in the systems that contained ascorbic acid. Ascorbic acid is known to have synergistic effects with other antioxidants by scavenging free radicals and regenerating oxidized antioxidants $[19,52]$. Thus, inclusion of antioxidants like ascorbic acid might have similar impacts on oleuropein stability as seen for the protection provided by its encapsulation, although more studies are required to understand the mechanisms involved here.

Liposomes have been studied previously as vehicles for bioactives to be incorporated into food matrices. As an example, the oxidation of ascorbic acid was significantly slowed in an encapsulated form in food matrices like apple juice and fermented milk [53]. Also, to increase their antioxidant capacity and delay lipid oxidation, antioxidant extracts have also been encapsulated in liposomes and incorporated into squid surimi gels [54].

\section{Conclusions}

The present study of the interactions of oleuropein with phospholipid membranes has shown that the oleuropein is located near to the membrane surface and can strongly interact with the phospholipid headgroups. This results in changes in the thermotropic behavior of the membranes, with fluidifying effects on the membrane at temperatures below the gel-to-liquid phase transition. Then at temperatures above this transition, the oleuropein can be partially inserted into the membranes, although remaining at a 'shallow' level, with the consequent increase in the order and packing of the phospholipid acyl chains. We have also shown that when oleuropein is actively encapsulated, these effects are stronger, which shows that a fraction of the oleuropein is effectively encapsulated, and results in a higher degree of interaction at the different levels of the multilamellae. Oleuropein also appeared to have significant radical-scavenging activity, and thus to act as an inhibitor of liposome peroxidation. To further investigate oleuropein as a functional ingredient, an OLE rich in oleuropein was encapsulated in liposomes. Oleuropein itself was encapsulated with a mean efficiency of $34 \%$, which indicated that optimization of these method or this process can be further investigated to improve the oleuropein encapsulation. However, liposome encapsulation was effective for a delay of oleuropein degradation at low $\mathrm{pH}$ (i.e., $\mathrm{pH}$ 2.0), and for the maintenance of oleuropein stability over long periods at refrigeration temperatures and at different pHs. This thus shows that this lipid encapsulation indeed provides a suitable carrier for OLE in food systems, such as beverages.

Acknowledgements This work was supported by the Italian Ministry of University and Research and has received funding from the European Union Horizon 2020 Research and Innovation Program under the Marie Skłodowska-Curie grant agreement $\mathrm{N}^{\circ} 713714$, and by the Slovenian Research Agency under the P4-0121 Programme and the CRP V4-1621 Project. The authors would like to thank OLEAFIT S.r.l (Isola del Gran Sasso, Italy) for kindly providing the olive leaf powder extract, and Nada Znidaršič for performing the transmission electron microscopy analysis.

Funding This work was supported by the Italian Ministry of University and Research and has received funding from the European Union Horizon 2020 Research and Innovation Programme under the Marie Skłodowska-Curie grant agreement $\mathrm{N}^{\circ} 713714$, and by the Slovenian Research Agency under the P4-0121 Programme and the CRP V4-1621 Project.

\section{Compliance with Ethical Standards}

Conflict of Interest The authors declare that they have no conflict of interest.

Open Access This article is licensed under a Creative Commons Attribution 4.0 International License, which permits use, sharing, adaptation, distribution and reproduction in any medium or format, as long as you give appropriate credit to the original author(s) and the source, provide a link to the Creative Commons licence, and indicate if changes were made. The images or other third party material in this article are included in the article's Creative Commons licence, unless indicated otherwise in a credit line to the material. If material is not included in the article's Creative Commons licence and your intended use is not permitted by statutory regulation or exceeds the permitted use, you will need to obtain permission directly from the copyright holder. To view a copy of this licence, visit http://creativecommons.org/licenses/by/4.0/.

\section{References}

1. C. Nediani, J. Ruzzolini, A. Romani, L. Calorini, Antioxidants 8, (2019)

2. I. Hassen, H. Casabianca, K. Hosni, J. Funct. Foods 18, 926 (2015)

3. B. Barbaro, G. Toietta, R. Maggio, M. Arciello, M. Tarocchi, A. Galli, C. Balsano, Int. J. Mol. Sci. 15, 18508 (2014)

4. A. Amini, M. Liu, Z. Ahmad, Int. J. Biol. Macromol. 101, 153 (2017)

5. Y. Liu, L.C. McKeever, N.S.A. Malik, Front. Microbiol. 8, 1 (2017)

6. L. Wang, C. Geng, L. Jiang, D. Gong, D. Liu, H. Yoshimura, L. Zhong, Eur. J. Nutr. 47, 235 (2008) 
7. Ú Catalán, L. Rubió, M.C. López de Las Hazas, P. Herrero, P. Nadal, N. Canela, A. Pedret, M.J. Motilva, R. Solà, Mol. Nutr. Food Res. 60, 2114 (2016)

8. A. Di Francesco, A. Falconi, C. Di Germanio, M.V.M. Di Bonaventura, A. Costa, S. Caramuta, M. Del Carlo, D. Compagnone, E. Dainese, C. Cifani, J. Nutr. Biochem. 26, 250 (2015)

9. M.A. Nunes, F.B. Pimentel, A.S.G. Costa, R.C. Alves, M.B.P.P. Oliveira, Innov. Food Sci. Emerg. Technol. 35, 139 (2016)

10. E. Ramírez, M. Brenes, P. García, E. Medina, C. Romero, Food Chem. 206, 204 (2016)

11. M.P. Carrera-González, M.J. Ramírez-Expósito, M.D. Mayas, and J. M. Martínez-Martos, Trends Food Sci. Technol. 31, 92 (2013)

12. M.C. López de las Hazas, C. Piñol, A. Macià, M.P. Romero, A. Pedret, R. Solà, L. Rubió, M.J. Motilva, J. Funct. Foods 22, 52 (2016)

13. M. Vinceković, M. Viskić, S. Jurić, J. Giacometti, D. Bursać Kovačević, P. Putnik, F. Donsì, F.J. Barba, and A. Režek Jambrak, Trends Food Sci. Technol. 69, 1 (2017)

14. S. Pimentel-Moral, M.C. Teixeira, A.R. Fernandes, D. ArráezRomán, A. Martínez-Férez, A. Segura-Carretero, E.B. Souto, Adv. Colloid Interface Sci. 260, 85 (2018)

15. B. Balanč, K. Trifković, V. Đorđević, S. Marković, R. Pjanović, V. Nedović, B. Bugarski, Food Hydrocoll. 61, 832 (2016)

16. K. Istenič, R. Cerc, Korošec, N. Poklar Ulrih, J. Sci. Food Agric. 96, $4623(2016)$

17. Q. Fei, D. Kent, W.M. Botello-Smith, F. Nur, S. Nur, A. Alsamarah, P. Chatterjee, M. Lambros, Y. Luo, Sci. Rep. 8, 1 (2018)

18. A. Saija, D. Trombetta, A. Tomaino, R. Lo Cascio, P. Princi, N. Uccella, F. Bonina, F. Castelli, Int. J. Pharm. 166, 123 (1998)

19. F. Paiva-Martins, M.H. Gordon, P. Gameiro, Chem. Phys. Lipids 124, 23 (2003)

20. N. Caturla, L. Pérez-Fons, A. Estepa, V. Micol, Chem. Phys. Lipids 137, 2 (2005)

21. D.D. Lasic, Liposomes: From Physics to Applications (Elsevier Science Ltd, Edinburgh 1993)

22. National Center for Biotechnology Information, PubChem Compd. Summ. CID 5287971 (2020). https://pubchem.ncbi.nlm.nih.gov/ compound/5287971. Accessed 31 July 2020

23. S. Perrett, M. Golding, W.P. Williams, J. Pharm. Pharmacol. 43, 154 (1991)

24. K.A. Riske, R.P. Barroso, C.C. Vequi-Suplicy, R. Germano, V.B. Henriques, M.T. Lamy, Biochim. Biophys. Acta - Biomembr. 1788, 954 (2009)

25. B.D. Balanč, A. Ota, V.B. Djordjević, M. Šentjurc, V.A. Nedović, B.M. Bugarski, N.P. Ulrih, Eur. J. Lipid Sci. Technol. 117, 1615 (2015)

26. V. Abram, B. Berlec, A. Ota, M. Šentjurc, P. Blatnik, N.P. Ulrih, Food Chem. 139, 804 (2013)

27. L.G.M. Basso, E.F. Vicente, E. Crusca Jr., E.M. Cilli, A.J. CostaFilho, Sci. Rep. 6, 37131 (2016)

28. R.D. Kaiser, E. London, Biochemistry 37, 8180 (1998)

29. L. Pérez-Fons, M.T. Garzón, V. Micol, J. Agric. Food Chem. 58, $161(2010)$
30. A.A. Jovanović, B.D. Balanč, A. Ota, P. Ahlin Grabnar, V.B. Djordjević, K.P. Šavikin, B.M. Bugarski, V.A. Nedović, N. Poklar, Ulrih, Eur. J. Lipid Sci. Technol. 120, 1800039 (2018)

31. E. Niki, Y. Yoshida, Y. Saito, N. Noguchi, Biochem. Biophys. Res. Commun. 338, 668 (2005)

32. G.P.C. Drummen, L.C.M. Van Liebergen, J. A. F. Op den Kamp, and J.A. Post, Free Radic. Biol. Med. 33, 473 (2002)

33. M. Laguerre, J. Lecomte, P. Villeneuve, Prog. Lipid Res. 46, 244 (2007)

34. N. Caturla, E. Vera-Samper, J. Villalaín, C.R. Mateo, V. Micol, Free Radic. Biol. Med. 34, 648 (2003)

35. C. Bonechi, A. Donati, G. Tamasi, A. Pardini, H. Rostom, G. Leone, S. Lamponi, M. Consumi, A. Magnani, C. Rossi, Biophys. Chem. 246, 25 (2019)

36. P. Trucillo, R. Campardelli, B. Aliakbarian, P. Perego, E. Reverchon, J. Supercrit. Fluids 135, 152 (2018)

37. J.J. Yuan, F.G.F. Qin, J.L. Tu, B. Li, Molecules 22, 1 (2017)

38. H. Tavakoli, O. Hosseini, S.M. Jafari, I. Katouzian, J. Agric. Food Chem. 66, 9231 (2018)

39. C. Sinico, C. Caddeo, D. Valenti, A.M. Fadda, A.R. Bilia, F.F. Vincieri, J. Liposome Res. 18, 83 (2008)

40. L. Funes, O. Laporta, M. Cerdán-Calero, V. Micol, Chem. Phys. Lipids 163, 190 (2010)

41. L. Funes, S. Fernández-Arroyo, O. Laporta, A. Pons, E. Roche, A. Segura-Carretero, A. Fernández-Gutiérrez, V. Micol, Food Chem. 117, 589 (2009)

42. A. Ota, K. Istenič, M. Skrt, N. Šegatin, N. Žnidaršič, K. Kogej, N.P. Ulrih, J. Food Eng. 229, 21 (2018)

43. M.R. Mozafari, C. Johnson, S. Hatziantoniou, C. Demetzos, J. Liposome Res. 18, 309 (2008)

44. D. Gmajner, P.A. Grabnar, M.T. Žnidarič, J. Štrus, M. Šentjurc, N.P. Ulrih, Biophys. Chem. 158, 150 (2011)

45. G. Altin, M. Gültekin-Özgüven, B. Ozcelik, J. Food Eng. 223, 91 (2018)

46. C. Romero, P. García, M. Brenes, Food Chem. 316, 126351 (2020)

47. E. Gikas, N. Papadopoulos, A. Tsarbopoulos, J. Liq. Chromatogr. Relat. Technol. 29, 497 (2006)

48. J. Connor, M.B. Yatvin, L. Huang, Proc. Natl. Acad. Sci. 81, 1715 (1984)

49. M. Lodzki, B. Godin, L. Rakou, R. Mechoulam, R. Gallily, E. Touitou, J. Control. Release 93, 377 (2003)

50. I. Gülseren, M. Corredig, J. Agric. Food Chem. 61, 3242 (2013)

51. R. Genç, M. Ortiz, C.K. O'Sullivan, Langmuir 25, 12604 (2009)

52. J.M. May, Z.C. Qu, S. Mendiratta, Arch. Biochem. Biophys. 349, 281 (1998)

53. L. Wechtersbach, N.Poklar Ulrih, B. Cigić, LWT - Food Sci. Technol. 45, 43 (2012)

54. D. Marín, A. Alemán, A. Sánchez-Faure, P. Montero, and M. C. Gómez-Guillén. Food Chem. 245, 525 (2018)

Publisher's Note Springer Nature remains neutral with regard to jurisdictional claims in published maps and institutional affiliations. 